\title{
EL TERCERMUNDISMO COMO PARADIGMA CIENTÍFICO EN AMÉRICA LATINA: EL PENSAMIENTO DE ORLANDO FALS BORDA ${ }^{1}$
}

\author{
The Third Worldism like scientific paradigm in \\ Latin America: Orlando Fals Borda's thinking
}

Germán Alburquerque*

\section{RESUMEN}

El artículo estudia el pensamiento tercermundista en general y el de Orlando Fals Borda en particular en su esfuerzo por constituir un paradigma científico sustentado en el Tercer Mundo. Esto quiere decir que se pensó que la ciencia social practicada en el Tercer Mundo -y con este concepto como referente de la investigación- originaría un nuevo paradigma que organizaría la totalidad del conocimiento. Se discute esta tentativa en el contexto de los estudios poscoloniales y decoloniales y a la luz de la reflexión que estas corrientes han elaborado sobre la hegemonía de la ciencia occidental en el mundo moderno.

Palabras clave: Tercermundismo, tercer mundo, ciencias sociales, paradigma científico, Orlando Fals Borda.

\footnotetext{
* Instituto de Estudios Avanzados, Universidad de Santiago de Chile. Santiago, Chile. Correo electrónico: german. alburquerque@usach.cl

${ }^{1}$ Este artículo es producto del Proyecto del Fondo Nacional de Desarrollo Científico y Tecnológico (FONDECYT) de Postdoctorado $\mathrm{N}^{\circ} 3110156$.

Artículo recibido el 19 de marzo de 2013. Aceptado el 14 de junio de 2013.
} 


\begin{abstract}
The article studies the third world thinking in general and the ideas of Orlando Fals Borda in particular, in the effort to constitute a scientific paradigm based on the Third World. It was thought that social science practiced in the Third World -and with this concept as referent for research- would originate a new paradigm that would organize the totality of the knowledge. This attempt is discussed in the context of the postcolonial and decolonial studies and in the light of the reflection that these currents have elaborated on the hegemony of western science in the modern world.
\end{abstract}

Keywords: Third world, third worldism, social sciences, scientific paradigm, Orlando Fals Borda.

\title{
INTRODUCCIÓN
}

En las últimas décadas ha cobrado relevancia el análisis del saber en relación con la expansión del capitalismo occidental y con el proceso de globalización que, a su vez, se vincula históricamente con el imperialismo y el orden colonial. Los estudios poscoloniales y luego decoloniales han puesto el acento en la clave cultural presente en la perpetuación de estructuras de dominación que no desaparecieron con la liberación política y jurídica alcanzada por los pueblos colonizados de los continentes periféricos. Gracias a esa labor se ha evidenciado la perniciosa acción del conocimiento sobre la realidad de los pueblos subdesarrollados, siendo en especial las ciencias sociales las más "sospechosas" de colaboración con los centros mundiales de poder localizados en Europa y Norteamérica. Como respuesta o reacción ha surgido la reclamación de una ciencia social, o en términos aún más amplios, de un paradigma o una epistemología, funcional a los intereses y necesidades del Sur y por supuesto no sujeta a las directrices provenientes del Norte, desde donde se ha dictado la marcha de la ciencia moderna. Sin embargo, tales esfuerzos no son inéditos ni mucho menos, es por ello que nos proponemos recuperar tentativas que entre los sesenta y los ochenta operaron en similar dirección aunque, de acuerdo a la sensibilidad rectora de la época, usaron como enseña Tercer Mundo 
o tercermundismo y no Sur o periferia. ${ }^{2}$

No cabe duda que los estudios poscoloniales o la opción decolonial han sido decisivos en la visibilización del problema del conocimiento. Dentro del grupo decolonial latinoamericano -integrado entre otros por Walter Mignolo, Aníbal Quijano, Fernando Coronil, Ramón Grosfoguel, Santiago Castro-Gómez, Nelson Maldonado-Torres, Zulma Palermo- ${ }^{3}$ quien se ha abocado en especial al papel de la ciencia social en la estructura de poder mundial es Edgardo Lander. Para este autor es vital cuestionar el conocimiento producido por el neoliberalismo y el pensamiento único, preguntarse cuáles son sus presupuestos, su concepción de mundo, los valores que lo inspiran. Y es que las consecuencias históricas que ha arrojado una determinada definición de ciencia han impactado de modo radical a los pueblos sometidos:

"La definición del conocimiento científico de Occidente como el único conocimiento objetivo y universal ha sido un mecanismo colonial-imperial que ha contribuido en forma crucial a los procesos de la destrucción o subordinación de toda otra opción del saber, y por ende, de toda otra opción de cultura y modo de vida, tanto en Europa como en el resto del planeta". ${ }^{4}$

Por eso Lander saluda el amplio y heterogéneo movimiento intelectual que por estos días ha sistematizado la crítica a lo que llama "saberes eurocéntricos coloniales modernos", y que ha ido más allá aun al buscar formas de conocimiento "que en lugar de naturalizar el actual orden global, permitan comprenderlo como orden histórico y por lo tanto superable". 5

En una tentativa similar a la nuestra, el venezolano capta en la historia cultural latinoamericana aquellas experiencias tendientes justamente a superar los

\footnotetext{
2 Por cierto, el debate acerca las consecuencias que sobre la humanidad entera han generado las ciencias sociales ha trascendido la reflexión académica para llegar a plantearse como un tema de discusión pública internacional. Primero fue la llamada Comisión Gulbenkian -auspiciada por la Calouste Gulbenkian Foundation- que tras una serie de conferencias entre 1994 y 1995 redactó un informe conocido como Abrir las ciencias sociales, obra firmada por el director de la comisión, Immanuel Wallerstein. Más tarde, en 1999, la UNESCO organizó en Budapest la "Conferencia Mundial sobre la Ciencia para el siglo XXI: un nuevo compromiso", de la cual emanó la "Declaración sobre la Ciencia y el uso del saber científico", que hacía votos por una ciencia al servicio de todos los pueblos.

${ }^{3}$ Ver Castro-Gómez, Santiago y Grosfoguel, Ramón (comps.). El giro decolonial: reflexiones para una diversidad epistémica más allá del capitalismo global. Bogotá: Siglo del Hombre Editores, 2007.

${ }^{4}$ En Lander, Edgardo. "Diálogos a través del Atlántico Sur: saberes hegemónicos y saberes alternativos". (c. 2000: 2). Disponible en: <http://bibliotecavirtual.clacso.org.ar/ar/libros/aladaa/lander.rtf>.

${ }^{5}$ Ibíd., 7.
} 
modelos exógenos para articular saberes propios y enfocados a las necesidades específicas del continente. Así destaca el movimiento intelectual/político que en las décadas del sesenta y setenta -en un contexto de agitación y lucha popularlogró crear un pensamiento crítico y reflexivo que "retomando algunos aspectos de lo que había sido descartado como producción ensayística y 'pre-científica', obvió la compartimentalización disciplinaria de la academia norteamericana, asumió perspectivas histórico estructurales y dejó a un lado la pretensión de la objetividad y el no-compromiso de la ciencia neutra, para asumir el conocimiento como instrumento de transformación". ${ }^{6}$

También con la mira puesta en la razón moderna, Walter Mignolo opone la razón postcolonial, que posee entre sus características primordiales la emergencia del "loci de enunciación de acciones sociales que surgen de los países del Tercer Mundo"7 y que no califican como ciencia si se siguen los moldes impuestos por el Primer Mundo. En otro trabajo Mignolo plantea una "epistemología de frontera" enraizada en distintos espacios del Tercer Mundo y en base a diferentes legados coloniales que se ofreciera al conocimiento y la civilización planetaria ${ }^{8}$. El occidentalismo, por el contrario, reservó solo para determinadas lenguas y localizaciones el dominio de la ciencia:

\footnotetext{
"Más allá de las ciencias sociales quedaron las prácticas de pensamiento. La reorganización de la producción del conocimiento, desde una perspectiva posoccidentalista, tendría que formularse en una epistemología fronteriza en la cual la reflexión (filosófica, literaria, ensayística) incorporada a las historias locales encuentra su lugar en el conocimiento desincorporado de los diseńos globales en ciencias sociales".
}

Quien parece aceptar el desafío planteado por la opción decolonial es el portugués Boaventura de Sousa Santos, cuyo libro Una epistemología del Sur ${ }^{10}$ lleva un título del todo significativo, pues su meta no es otra que dotar a los pueblos periféricos y víctimas de la globalización de un nuevo paradigma.

\footnotetext{
${ }^{6}$ Ibíd., 4.

${ }^{7}$ Mignolo, Walter. "Herencias coloniales y teorías postcoloniales". En Beatriz González Stephan (ed.). Cultura y Tercer Mundo: 1. Cambios en el Saber Académico. Caracas: Nueva Sociedad, (1996): 118.

${ }^{8}$ Mignolo, Walter. "Posoccidentalismo: las epistemologías fronterizas y el dilema de los estudios (latinoamericanos) de áreas”, Revista Iberoamericana LXII/176-177, julio-diciembre, (1996): 688-689.

${ }^{9}$ Ibíd., 692.

${ }^{10}$ Santos, Boaventura De Sousa. Una epistemología del Sur: la reinvención del conocimiento y la emancipación social. México: Siglo XXI-CLACSO, 2009.
} 
Para Santos ya es hora de consumar la crítica al modelo de racionalidad occidental que desde al menos dos siglos se extiende sin contrapeso, en circunstancias que es insuficiente para la comprensión cabal del orbe no occidental. Además ello ha comportado hondos perjuicios sobre las sociedades periféricas:

"Como el conocimiento científico no se encuentra distribuido de una forma socialmente equitativa, sus intervenciones en el mundo real tienden a ser las que sirven a los grupos sociales que tienen acceso a este conocimiento. En última instancia, la injusticia social descansa en la injusticia cognitiva". ${ }^{11}$

El libro al que nos referimos reúne distintos ensayos y artículos por lo que es posible extraer más de una sola formulación. Así, por una parte el autor invita a reemplazar la razón indolente, como llama a la racionalidad occidental, por la razón cosmopolita, fundada en tres procedimientos "metasociológicos", que son, a saber, la sociología de las ausencias, la sociología de las emergencias y el trabajo de traducción, que en conjunto configuran un sistema complejo que no cabe detallar en este trabajo. ${ }^{12} \mathrm{Y}$ por otra, apuesta por una ecología de saberes que corrija la ignorancia e incapacidad de la ciencia occidental frente a saberes no científicos de otras áreas y propenda hacia una diversidad epistemológica global. No se trata de desacreditar el conocimiento científico, sino de realzar otros modos de conocer para darles un uso contrahegemónico consistente "por un lado, en explorar la pluralidad interna de la ciencia, eso es, prácticas científicas alternativas que han sido hechas visibles por epistemologías feministas y postcoloniales y, por otro lado, en promover la interacción e interdependencia entre conocimientos científicos y no-científicos". 13

Para Maritza Montero la tarea ya está realizada, y en la propia América Latina, pues en las últimas décadas se han decantado formas alternativas de conocimiento que permiten, hacia fines del siglo XX, declarar la existencia de un "modo de ver el mundo, de interpretarlo y de actuar sobre él" que constituye un

\footnotetext{
${ }^{11}$ Ibíd., 115.

12 "La comprensión del mundo [occidental] y la forma cómo ella crea y legitima el poder social tiene mucho que ver con concepciones del tiempo y de la temporalidad. [...] La característica más fundamental de la concepción occidental de la racionalidad es el hecho de, por un lado, contraer el presente y, por otro, expandir el futuro. La contracción del presente, originada por una peculiar concepción de la totalidad, consiste en transformar el presente en un instante huidizo, atrincherado entre el pasado y el futuro. Del mismo modo, la concepción lineal del tiempo y la planificación de la historia permitieron expandir el futuro indefinidamente" (Ibíd., 100).

${ }^{13}$ Ibíd., 186.
} 
episteme con el cual el continente "está ejerciendo su capacidad de ver y hacer desde una perspectiva Otra, colocada al fin en el lugar de Nosotros". ${ }^{14}$ Los principios que sustentan este nuevo paradigma serían: una concepción de comunidad, de participación y de creación del saber popular que conduce a un episteme de relación; el concepto de liberación que, por medio de la praxis, echa a andar la conciencia y el sentido crítico que derivan en la desnaturalización de las formas tradicionales de "aprehender-construir-ser en el mundo"; la redefinición del papel del investigador social que conlleva el reconocimiento de un sujeto-objeto que es a la vez actor social y generador de conocimiento; la pluralidad epistémica resultante del carácter histórico, en construcción y relativo del conocimiento; la perspectiva de la dependencia y de la resistencia; y la constante revisión de métodos, con las transformaciones que provoca. La autora subraya las fuentes más fecundas que han concurrido en la constitución de este paradigma latinoamericano: la teología y la filosofía de la liberación y, entre las personalidades, Paulo Freire, Alejandro Moreno y Orlando Fals Borda.

Teniendo en cuenta este panorama actual procederemos a buscar las iniciativas que en el pasado entregaron ya similares orientaciones. Pero antes de entrar de lleno en ello realizaremos algunos deslindes para acotar mejor nuestro objeto y evitar confusiones.

Primero precisemos qué entendemos por tercermundismo. Si bien se origina en un concepto creado por la ciencia social europea, el tercermundismo pronto se convirtió en estandarte identitario que interpeló a los pueblos de África, América Latina y Asia y que dio lugar a una ideología, entendida ésta como un conjunto -no sistemático- de ideas acerca de la realidad del Tercer Mundo, de su formación y del modo de superar su deficitario estado. El tercermundismo derivó también en una sensibilidad que tińó con su color el ambiente cultural de los sesenta y setenta y, para terminar, adquirió ribetes de paradigma sobre todo en el área de las ciencias sociales.

El tercermundismo, en consecuencia, generó ideas, discursos, emociones

\footnotetext{
${ }^{14}$ Montero, Maritza. "Paradigmas, conceptos y relaciones para una nueva era. Cómo pensar las Ciencias Sociales desde América Latina”, mimeo (1998), citado por Lander, Edgardo. "Ciencias sociales: saberes coloniales y eurocéntricos". En Edgardo Lander (ed.). La colonialidad del saber: eurocentrismo y ciencias sociales. Perspectivas latinoamericanas. Buenos Aires: CLACSO, (2000): 9-10. Cito la versión disponible en: <http://bibliotecavirtual.clacso.org.ar/ar/libros/lander/lander1.rtf>.
} 
y análisis. Dentro de estos, un primer intento a rescatar es la denuncia de la dependencia científica del Tercer Mundo. A la dependencia económica, política, militar, etc., debía sumarse el atraso de la ciencia -en general-que en los continentes periféricos, de partida, no recibía los fondos suficientes para su desarrollo, quedando supeditada a las transferencias científicas y tecnológicas provenientes del centro. De esta postura se deduce que el Tercer Mundo debe financiar la investigación científica para poder competir en condiciones más equilibradas y así acercarse al tan deseado desarrollo.

Un paso más audaz fue la valoración del conocimiento originario del Tercer Mundo, un conocimiento no necesariamente científico o al menos no regido por la concepción occidental de ciencia. Se trataba de ponderar los adelantos que los pueblos del Tercer Mundo habían logrado con anterioridad a la llegada de la conquista europea, y que el conocimiento científico tradicional había desdeñado como pensamiento precientífico o primitivo, y que en realidad eran ajenos a los moldes de la ciencia europea pero no por eso menos válidos. Se reivindicaban así formas no tradicionales de conocimiento, una tendencia que iría creciendo y adquiriendo un estatus cada vez mayor. Cabe hacer la salvedad de que en este esfuerzo participaron no solo especialistas del Tercer Mundo sino también del mundo desarrollado.

Es desde ésta área, el Primer Mundo, que emergerá otra línea cercana a lo que nos interesa. Me refiero a los Third World Studies, llevados a cabo por estudiosos estadounidenses e ingleses, en su mayoría, que al ocuparse de regiones específicas del Tercer Mundo fundaron esta subdisciplina que, por cierto, no perseguía cambiar paradigmas científicos sino la especialización profesional. Los Third World Studies fueron objeto de la aguda crítica de Carl Pletsch, quien estableció una suerte de división internacional del trabajo en el campo de las ciencias sociales. ${ }^{15}$ Así, fue la antropología la disciplina que se ocupó del Tercer Mundo -al menos hasta que sus países alcanzaban cierto grado de modernización, en cuyo caso pasaban a interesar a la sociología, la politología y la economía- así como los estudios de área se ocuparon del orbe socialista. Por otra parte, dichos estudios obedecieron a la necesidad de conocer la realidad de unos pueblos que en el juego geopolítico de la época se convirtieron en un preciado botín de guerra a

\footnotetext{
${ }_{15}$ Pletsch, Carl. "The Three Worlds, or the Division of Social Scientific Labor, circa 1950-1975", Comparative Study of Society and History (1981): 565-590.
} 
disputar con el bloque soviético. En el fondo, a lo que apunta Pletsch es al lugar de enunciación de los discursos científicos, en el sentido que es el Primer Mundo el que dictamina no solo qué disciplinas son las apropiadas, sino la misma división del planeta en tres sectores estereotipados.

Todo lo anterior está lejos de constituir un nuevo paradigma científico tercermundista, aunque sin duda contribuyó a la creación de un clima adecuado para el surgimiento del mismo. En lo que sigue nos referiremos a los intentos más serios que en esa dirección afloraron en América Latina entre los años sesenta y ochenta. Ello no implica que en el resto del Tercer Mundo, en Asia y África, no hayan aparecido iniciativas semejantes. ${ }^{16}$

\section{EL TERCERMUNDISMO COMO PARADIGMA: ORLANDO FALS BORDA (Y ALGO MÁS)}

Que el tercermundismo haya sido concebido como un paradigma científico quiere decir que el Tercer Mundo fue considerado el prisma que permitía observar y entender el mundo social y en alguna medida también el natural; el eje ordenador del objeto a estudiar, de las preguntas a formularse, de los procedimientos empleados y de la interpretación de los resultados. ${ }^{17} \mathrm{Tal}$ aserción se fundaba en ciertos principios: que la investigación científica concentrada en los genuinos problemas de los países subdesarrollados adquiere características únicas que determinan una nueva metodología que es a su vez aplicable a escala mundial; que las revoluciones acaecidas en el Tercer Mundo ofrecen una praxis replicable en otras regiones, una praxis que es verdadera en tanto proviene del éxito revolucionario; que el Tercer Mundo requiere producir sus propios conceptos y

\footnotetext{
${ }^{16}$ Sin embargo, como una muestra de que estas preocupaciones encuentran eco en otras regiones, podemos señalar un artículo de Paula Durán donde reseña algunas ideas de Abdelkebir Khatibi (citando su obra Magreb Pluriel, 1983) en orden a descolonizar la sociología árabe mediante dos vías, primero, deconstruyendo los conceptos importados del discurso sociológico occidental, y segundo, criticando los discursos que las sociedades árabes han elaborado sobre ellas mismas (Durán Monfort, Paula. "Por una descolonización de las ciencias sociales. Alternativas antropológicas desde el Magreb". En Aitzpea Leizaola y Jone Miren Hernández (coords.). Miradas, encuentros y criticas antropológicas. San Sebastián: Ankulegi Antropologia Elkartea, (2008): 177. En tanto, Denos Kouassi, desde Costa de Marfil, se cuestiona el papel de la antropología tercermundista y hace un llamado a constituir una asociación de antropólogos del Tercer Mundo para así "influir mejor en los proyectos de desarrollo que se proponen en el tercer mundo o para el tercer mundo" (Kouassi Koffi, Denos. "Antropología y tercermundismo. Una propuesta de ruptura", Gazeta de antropología 9 (1992): 11.

${ }^{17}$ Tomamos el concepto paradigma en su connotación epistemológica y teniendo en cuenta la definición que entrega Khun en La estructura de las revoluciones cientificas: lo que se debe observar y escrutar; el tipo de interrogantes que se supone hay que formular para hallar respuestas en relación al objetivo; cómo tales interrogantes deben estructurarse; y cómo deben interpretarse los resultados de la investigación científica.
} 
categorías liberándose así de los rígidos esquemas de la ciencia occidental; que una auténtica ciencia social enunciada desde el Tercer Mundo no puede resultar sino en algo teóricamente nuevo y alternativo a la ciencia social occidental.

En América Latina, un antecedente del tercermundismo en su dimensión epistémica lo constituye el liberacionismo, primero desenvuelto en pedagogía, teología y filosofía, pero luego cultivado en sociología, aunque con menos fuerza. Se habló de una sociología de la liberación concentrada en las agudas crisis de los pueblos dependientes que fuera tanto estudio como acción o intervención. El principal mentor del tercermundismo como paradigma, como veremos a continuación, tuvo como punto de partida esta opción.

Si bien el tercermundismo en América Latina fue una sensibilidad que incluyó más o menos consensualmente la conciencia de que la ciencia social, tal como se conocía, se veía afectada por el nuevo referente y que por ende debía reformarse, lo que haremos aquí será concentrarnos en las propuestas más elaboradas sobre la materia.

El sociólogo colombiano Orlando Fals Borda (1925-2008) debe ser considerado el gran articulador del tercermundismo como paradigma científico. No fue un descubrimiento temprano ni súbito, sino que fue una construcción que le tomó años. En efecto, si bien se sintió interpelado por el Tercer Mundo desde los años sesenta, veremos que en sucesivas entregas fue moldeando con cada vez mayor claridad su concepto de ciencia tercermundista.

El primer paso lo dio en 1970, cuando publicó Ciencia propia y colonialismo intelectual, compilado de artículos, ensayos, reelaboraciones y textos inéditos que en conjunto conformaron una sistematización teórica de un investigador volcado a lo empírico pero siempre llamado por la alta teoría. Estas facetas, la de investigador en terreno y la de teórico de la sociología o de la ciencia social, se combinaron en él de modo inextricable.

La más significativa proclama del volumen era, como anunciamos, la apuesta por una "sociología de la liberación", un paso, aunque lógico y previsible, importante para una disciplina que solo en los cincuenta y sesenta alcanzó, en 
América Latina, rigor científico. La sociología en que se desenvolvía Fals Borda estaba tensionada por distintas corrientes -la sociología de la modernización; la de corte positivista y empírica proveniente de Estados Unidos; la de inspiración marxista y militante; la sociología del desarrollo, etc.-, por lo cual anunciar una sociología de la liberación hacia 1970 suponía superar estos conflictos y emparentar la disciplina con una liberación que era juzgada como inminente no solo en América Latina sino también en el resto del Tercer Mundo. ${ }^{18}$

Sin embargo, el pensamiento de Fals Borda, hasta este punto, denotaba un horizonte esencialmente latinoamericano. Su preocupación general eran los países subdesarrollados, y aunque adoptaba una explícita postura del Tercer Mundo, su pensamiento aún se restringía a la realidad particular de su continente. Dentro de ese marco, y abocado a la constitución de una sociología de la liberación, toma como punto de partida la necesidad de fundar una ciencia propia, autónoma, concebida por y para los latinoamericanos, expresión de sus conflictos, soberana de sus temas de investigación y en rebelión frontal con la sociología europea y norteamericana. Así invitaba a "trabajar arduamente con nuestros materiales y realidades, tratando de articular nuestras respuestas con fórmulas, conceptos y marcos de referencia de aquí mismo" ${ }^{19}$

Esta ciencia "rebelde y subversiva" debía conquistar la independencia cultural, técnica y científica que a su vez conduciría a la independencia política y económica de América Latina. Y es que la sociología no podía ser simple espectadora de los cambios que estremecían el continente, debía ser parte activa, creadora y "liberadora", utilizando "el método científico para describir, analizar y aplicar el conocimiento para transformar la realidad, trastocar la estructura de poder y de clases que condiciona esa transformación y poner en marcha todas las medidas conducentes a asegurar una satisfacción más amplia y real del pueblo”. ${ }^{20}$

Fals Borda creía en una sociología comprometida y reconcentrada en los

\footnotetext{
${ }^{18}$ En 1975 el argentino Pedro Negre Rigol también haría el cruce entre sociología de la liberación y Tercer Mundo, llegando a proposiciones similares. Llama la atención que este autor mencione apenas un par de veces a Fals Borda y no cite ninguno de sus escritos. Ver Negre Rigol, Pedro. Sociología del Tercer Mundo. Una introducción a sus problemas sociológicos. Buenos Aires: Paidós, 1975.

${ }^{19}$ Fals Borda, Orlando. Ciencia propia y colonialismo intelectual. México: Nuestro Tiempo, (1973): 19.

${ }^{20}$ Ibíd., 23.
} 
fenómenos de cambio, conflicto y crisis, manifestaciones de las fuerzas que querían acabar con la dependencia y la explotación y con sus instigadores, el imperialismo y el colonialismo. Pero de esta lucha emergería un aporte sustantivo y universal al saber sociológico:

"La ciencia social verá el surgimiento de un nuevo e interesante conjunto de teorías y conceptos construidos alrededor del proceso político liberador, en respuesta a la superación de la actual crisis... Esta 'sociología de la liberación' sería un acto de creación científica que satisfaría al mismo tiempo los requisitos del método y de la acumulación del conocimiento científico... Teoría y práctica, idea y acción se verían así sintetizadas”. ${ }^{21}$

Se lanzaba a combatir la imitación de modelos y temas de la ciencia social occidental, por eso llamaba a romper las cadenas y menguar el servilismo y colonialismo intelectual, para examinar, en cambio, "los procesos y mecanismos de la toma del poder por las clases populares, la búsqueda de nuestra razón de ser y una explicación propia de nuestras realidades". ${ }^{22}$

Si bien el concepto Tercer Mundo aparece en su discurso, declarándose parte del mismo, en su esquema el tercermundismo es marginal, casi decorativo, lo cual se aprecia en estas líneas que denotan cierta distancia. Comentando la necesidad de conocer otras realidades internacionales, admitía que esto "lleva también a vincularnos mediante la investigación y la asistencia técnica a los países del Tercer Mundo, entre los cuales es meritorio destacar a los del África, que son excelentes laboratorios sociales, casi todos en plena y acelerada evolución”. ${ }^{23}$ Agregaba que los africanos prefieren trabajar con técnicos de países subdesarrollados porque enfrentan problemas comunes como la falta de estadística, el analfabetismo y el atraso agrícola-técnico. Ciertamente esta es una afirmación de identidad tercermundista, pero es también la afirmación de que África es un otro, que se nos parece, pero que no es todavía un nosotros.

Ya en 1980 la presencia del Tercer Mundo adquiere mayor cuerpo. No es menor, en ese sentido, que el título de un artículo lleve aquel concepto. Me refiero a "Una perspectiva para las ciencias sociales del Tercer Mundo", en donde, además

\footnotetext{
${ }^{21}$ Ibíd., 46.

${ }^{22}$ Ibíd., 29.

${ }^{23}$ Ibíd., 83.
} 
de usar con más frecuencia el término, ahondaba en la vinculación entre ciencia y Tercer Mundo, apuntando a la especificidad que la sociología adopta en los países periféricos, sobre todo mediante lo que llama “investigación-acción radical”, la cual "responde a la necesidad de cierto tipo de conocimiento científico comprometido con determinadas clases sociales, a fin de acelerar procesos de cambio estructural y revolucionario". ${ }^{24}$ Ello ha significado que europeos y estadounidenses sigan con atención este esfuerzo.

Lo insinuado por Fals Borda se materializará hacia 1990, cuando aparece en Nueva Sociedad de Caracas la trascripción de un discurso realizado poco antes en una universidad colombiana, que no se contentaba con llevar "Tercer Mundo" en el título, sino que lo erigía como referente principal y centro de la reflexión. ${ }^{25}$ Sostengo que en este escrito Fals Borda lleva a su máxima expresión la constitución del tercermundismo como referente científico, un paradigma básico o blando, en construcción, decantado principalmente por la experiencia de la investigación social en el escenario de los países tercermundistas.

Es interesante constatar que Fals Borda pensaba el devenir de la ciencia social como un proceso global y sistémico que involucraba a los cinco continentes y en donde los investigadores se situaban en continuo diálogo. Esto era crucial para sentar la fuerza de la ciencia social de los países del Tercer Mundo pues en ella radicaba el progreso de la disciplina en general, desplazando de esta posición de vanguardia a la ciencia de los países desarrollados. En este ámbito Fals Borda certificaba un agotamiento que hundía sus raíces en la crisis existencial e ideológica que recorría el Primer Mundo y que se explicaba "sea por las proclividades autoobjetivantes de la ciencia y la técnica modernas desarrolladas allí, sea porque hoy surgen amenazas serias para la supervivencia de todo el género humano relacionadas con los avances inconsultos de esa misma ciencia euroamericana fetichizada y alienante". ${ }^{26}$

\footnotetext{
${ }^{24}$ Fals Borda, Orlando. "Una perspectiva para las ciencias sociales del Tercer Mundo", Comercio Exterior 30/7 (1980): 674. ${ }^{25}$ Fals Borda, Orlando. "El Tercer Mundo y la reorientación de las ciencias contemporáneas", Nueva Sociedad 107, mayojunio 1990 (paginación según versión en internet).

${ }^{26}$ Ibíd., 2.
} 
Así las cosas, los científicos de los países avanzados de las zonas templadas -como los llama el colombiano- han volcado su atención al quehacer intelectual de los países "tropicales y subtropicales", en donde buscarían una savia nueva y pura que revertiría el estancamiento en que se hallan. Pero para que se interesen en la ciencia tercermundista primero debieron observar con respeto la realidad de los pueblos subdesarrollados para luego asumir una posición crítica respecto a la ciencia propia, tomando conciencia de sus vicios y debilidades. Investigadores críticos del Primer y Tercer Mundo establecen pues una alianza, aunque ahora, a diferencia del pasado, son los tercermundistas los que proponen y los primermundistas los que siguen. Luego, los últimos progresos de la ciencia social "se han realizado dentro de un marco crítico común, que invita a retar políticamente a las instituciones del poder formal, así en los países dominantes como en los dependientes" ${ }^{27}$ Nótese cómo las disciplinas humanas tienen como meta un objetivo político y subversivo, en consonancia con la "sociología de la liberación" esbozada por Fals Borda hacía dos décadas.

Reconocía con júbilo a aquellos científicos del Primer Mundo que se han rebelado y orientado hacia el Sur y que han manifestado "un querer sentir y comprender empáticamente" los valores de nuestras sociedades, aunque tampoco se le escapaba que tal esfuerzo obedecía en buena medida a la nostalgia con que apreciaban la resistencia de los "indígenas y campesinos analfabetas y explotados del Tercer Mundo ante los daños y perjuicios del desarrollo capitalista y de la racionalidad instrumental". ${ }^{28}$ Desde los países avanzados se ha recuperado la problemática del Tercer Mundo, aplicando sus enfoques y temas a su realidad particular. De esta manera ha ido mutando el ordenamiento tradicional del progreso técnico y científico, definido por la influencia y el traspaso NorteSur, al punto que Fals Borda vislumbra -aun de modo parcial- la reversión de tal dirección, pasando a ser el Sur el que alimenta epistémicamente al Norte, creándose en definitiva “olas de convergencia temática”. Éstas -profundizaba el colombiano- no apuntan al mero conocimiento, sino que se aplican a la realidad con la intención de cambiarla, siguiendo la "vieja consigna de conocer para poder actuar bien y transformar mejor"; en ese sentido presenciaríamos el despunte de "una hermandad universal comprometida políticamente contra sistemas

\footnotetext{
${ }^{27}$ Ibíd., 1 y 2.

${ }^{28}$ Ibíd., 5.
} 
dominantes, una hermandad conformada por colegas intensamente preocupados por la situación social, política, económica y cultural de todos nosotros". ${ }^{29}$

Por supuesto, más que la actitud pionera de los investigadores de los países centrales, ha sido el desarrollo que ha experimentado la ciencia en el Tercer Mundo lo que ha generado el nuevo orden del conocimiento científico mundial, constituyéndose en una "contracorriente intelectual autonómica" que está incidiendo en círculos políticos y académicos de los países del norte. Pensaba que había surgido un contingente de investigadores sociales y políticos que desafiaba el statu quo local pero que inspiraba la misma lucha en otras sociedades. Ahora, ¿por qué las ciencias sociales del Tercer Mundo han tomado la delantera y superado a las restantes?

Como hemos ya apuntado, el paradigma tercermundista se construye a partir de la investigación empírica, de la práctica, y se ha beneficiado del cúmulo de la producción científica de esta parte del mundo. A partir de esa experiencia ha logrado elaborar teorías y métodos propios que han contestado anteriores interpretaciones "exogenéticas o eurocéntricas". Quizá la quinta esencia de este naciente paradigma se localice en el modo vivencial de obtener y desarrollar conocimiento. Los mismos europeos y norteamericanos estarían ávidos de esta vivencia, imposible ya de encontrar allí, pero abundante en los pueblos subdesarrollados, "donde existe aún la praxis original no destruida por el capitalismo industrial: aquí en América Latina, en África, en Oceanía”. ${ }^{30}$ En la tarea de alcanzar "formas satisfactorias de sabiduría, razón y poder" era necesario abrirse a todas las expresiones intelectuales que muchas veces han sido reprimidas por universidades y gobiernos:

"Tanto en Europa como en la India y en Colombia buscamos métodos frescos y alegres de organización popular, diferentes de los impuestos por los dogmas [...] Y salen voces 'bacanas' y luces correctivas desde nuestros países subdesarrollados que iluminan la potencialidad creadora de los azares de las luchas, de la espontaneidad y de la intuición de las masas, para ir organizando movimientos regionales sociales y políticos independientes". ${ }^{31}$

\footnotetext{
${ }^{29}$ Ibíd., 5.

${ }^{30}$ Ibíd., 2 y 3.

${ }^{31}$ Ibíd., 11.
} 
En un plano concreto, Fals Borda entrega un repertorio de aquellos pensadores o escuelas que estarían cristalizando la influencia del Sur en el Norte en el plano epistémico de las ciencias sociales y humanas e incluso de la naturaleza. Así mencionaba los "escritores historiadores latinoamericanos", como Eduardo Galeano y Alejo Carpentier, dada su universalidad; la "dialógica moderna", cuyo adalid era Paulo Freire y que había suscitado un cambio en la educación de adultos a escala global; las teorías de la dependencia, con exponentes como Samir Amin, Fernando H. Cardoso, Enzo Faletto, Celso Furtado y André Gunder Frank; las teorías de la CEPAL u otras que han cuestionado los fundamentos de la economía tradicional, destacando la crítica de los "economistas descalzos" (por ejemplo, Manfred Max-Neef); la "propuesta praxiológica de la subversión moral" iniciada, según el colombiano, entre "las gentes de nuestras islas y montañas" y bajo la égida de Camilo Torres y Ernesto Guevara; la teología de la liberación, con Gustavo Gutiérrez y Leonardo Boff, que ha trastocado la iglesia universal; el rescate de los "grupos sin historia" comandado por "bengalíes, hindúes y srilanqueses"; las investigaciones provenientes de todos los continentes periféricos, incluido Oceanía, que han renovado el esclerosado marxismo europeo (Bartra, Stavenhagen, González Casanova, Benarjee, Taussing, Mustafá); las teorías del estado y la democracia nacidas en el Cono Sur de América Latina (N. Lechner y G. O’Donnell). Por último consigna el aporte de los hindúes a la física cuántica. ${ }^{32}$

Concluye el sociólogo colombiano con la esperanza de que la autonomía científica del Tercer Mundo fuera la piedra angular de su definitiva liberación frente al colonialismo, el imperialismo y el capitalismo.

Una visión más radical y política fue la que sostuvieron el sociólogo costarricense Miguel Sobrado y al etnólogo polaco Z. Martin Kowalewski, autores -en colaboración- de Antropología de la guerrilla (Hacia la ciencia social del Tercer Mundo). En esta obra establecían una relación directa entre la práctica política y un conocimiento científico-social orientado a superar el pensamiento marxista clásico, juzgado insuficiente para los pueblos subdesarrollados debido a que fue concebido para la realidad europea: "Consideramos que la realidad social de estas zonas periféricas del capitalismo mundial hay que examinarla en las categorías conceptuales que en la práctica ya consiguieron afirmarse como más o menos

32 Ibíd., 7 y 8. 
adecuadas ante todo para el Tercer Mundo". ${ }^{33}$ Por consiguiente la nueva teoría se derivaría de la práctica concreta, de las correctas interpretaciones y soluciones puestas en ejercicio en las nacientes sociedades. La ciencia social a la que aspiraban debía ser pensada desde y para el Tercer Mundo, bajo el entendido de que aquello que era adecuado para un país periférico de América Latina lo sería también para una nación africana o asiática (o viceversa).

En el título se aludía a la guerrilla porque de la praxis revolucionaria -no solo de la toma del poder, sino también de su ejercicio- se extraería finalmente, para las ciencias sociales, una verdad absoluta. Por ello invitaban a tomar conciencia de los avances de la investigación que han surgido del proceso de transformación y de ruptura revolucionaria puesto en marcha en los distintos países liberados. De la acumulación de experiencias positivas se llegaría a una suma de este flamante saber que, sin componer un todo coherente, estaría constituido por "el conjunto de ideas o conceptos que emergen en la reflexión de los hombres de la acción revolucionaria en el tercer mundo sobre sus propias actividades empíricas, o sobre las que dirigen en función de las mutaciones estructurales de sus sociedades; por el conjunto de conocimientos comunicados para guiar teóricamente la acción o simplemente para objetivizar las experiencias subjetivas acumuladas, o las directamente vividas en tal o cual acto práctico". ${ }^{34}$

\section{CONCLUSIONES}

1. Debemos advertir que los autores tratados no usan el concepto paradigma; es desde nuestra perspectiva que hemos reconocido en el pensamiento tercermundista toda una elaboración sobre la ciencia que -nos parece- avanzaba hacia la constitución de un nuevo paradigma. Sin duda la tentativa no llegó a concretarse, en el sentido de que, según lo que se entiende por paradigma científico, no se extendió por la comunidad científica entera, aunque sí obtuvo bastante consenso en cierta fracción del campo intelectual latinoamericano de la época, en especial durante los sesenta y setenta. Sí debemos subrayar que el Tercer Mundo fue un concepto catalizador que dinamizó la reflexión sobre los alcances y las posibilidades de la ciencia en la tarea de transformar el mundo.

\footnotetext{
${ }^{33}$ Kowalewski, Zbigniew Zbigniew Martin y Sobrado, Miguel. Antropología de la guerrilla (Hacia la ciencia social del Tercer Mundo). Caracas: Nueva Izquierda, (1971): 7.

${ }^{34}$ Ibíd., 11.
} 
Queremos expresar que sin el tercermundismo no se habrían generado las ideas de Fals Borda, Sobrado y compañía que apuntaban al mismo objetivo que, con posterioridad, los estudios poscoloniales y la decolonialidad pusieron en la primera línea de la discusión. Allí radica el valor del paradigma científico tercermundista: un hito precursor, una formulación que abrió paso a una línea de pensamiento muy fecunda y con gran vigencia. Si la aspiración es menguar la hegemonía de la ciencia occidental y permitir la emergencia de modalidades alternativas de saber, lo planteado por el tercermundismo conviene no ser olvidado.

2. Lo anterior no impide una lectura crítica de las ideas estudiadas. En ese plano, ¿tuvo el paradigma tercermundista suficiente riqueza científica como para realizarse? Creemos que las formulaciones pecaron, en mayor o menor medida, de inocencia. Resulta difícil aceptar que la investigación científica, por el mero hecho de ser practicada en el Tercer Mundo, adquiriría un nuevo estatuto, como parece indicar Fals Borda. Es posible, en efecto, que de la experiencia de los cientistas sociales en los países subdesarrollados surgieran nuevas técnicas, nuevas preguntas y nuevos resultados; como también lo es que tales progresos pudieran transmitirse y ser recogidos por la ciencia social del Centro... Pero, ¿se está fundando una ciencia nueva?, ¿se está dando un giro epistémico? Más débil aun asoma lo planteado por Antropología de la guerrilla, que consagra la práctica revolucionaria como fuente de verdad para las ciencias sociales. El hecho de que una medida sea exitosa en un país revolucionario de la periferia, y por lo tanto aplicable a otras regiones no supone de por sí una superación del marxismo ni tampoco la creación de nuevos conceptos y categorías. Por otra parte, Fals Borda destaca los aportes de las ciencias sociales, de la literatura, de la lucha política, de la física cuántica, en fin, del Tercer Mundo para el resto del planeta, y de ello concluye que se estaría invirtiendo el flujo de la transmisión del conocimiento, que ahora sería Sur-Norte. Su afirmación es un paso importante y la semilla de lo que podrían ser investigaciones muy valiosas en una problemática donde quedan muchas cosas por esclarecer. Pero es discutible si tales contribuciones representan formas realmente alternativas de conocimiento, capaces de reformular la ciencia occidental, aunque también debemos preguntarnos si ello es de verdad posible. Me inclino a pensar que desde hace mucho tiempo asistimos a una constante dialéctica entre saberes de distinto origen, y que es así como la razón occidental ha ido adquiriendo, lentamente, elementos de otras tradiciones epistémicas, así 
como éstas han sido "contaminadas" por aquella, lo cual no significa ignorar que la primera ejerce una hegemonía destructiva sobre las segundas, hegemonía que es preciso detener o al menos moderar.

\section{REFERENCIAS}

Castro-Gómez, Santiago y Grosfoguel, Ramón (comps.). El giro decolonial: reflexiones para una diversidad epistémica más allá del capitalismo global. Bogotá: Siglo del Hombre Editores, 2007.

Durán Monfort, Paula. "Por una descolonización de las ciencias sociales. Alternativas antropológicas desde el Magreb”. En Aitzpea Leizaola y Jone Miren Hernández (coords.). Miradas, encuentros y criticas antropológicas. San Sebastián: Ankulegi Antropologia Elkartea, 2008.

Fals Borda, Orlando. Ciencia propia y colonialismo intelectual. México: Nuestro Tiempo, 1973.

Fals Borda, Orlando. "Una perspectiva para las ciencias sociales del Tercer Mundo", Comercio Exterior 30/7, julio, 1980.

Fals Borda, Orlando. "El Tercer Mundo y la reorientación de las ciencias contemporáneas”, Nueva Sociedad 107, mayo-junio, 1990.

Guerrero Barón, Javier y García Sánchez, Bárbara. "La Ciencias Sociales y la invención del Tercer Mundo: A propósito de la obra académica de Orlando Fals Borda (I)", Revista Historia de la Educación Latinoamericana 12, 2009.

Kouassi Koffi, Denos. "Antropología y tercermundismo. Una propuesta de ruptura". Gazeta de antropología 9, 1992.

Lander, Edgardo. "Ciencias sociales: saberes coloniales y eurocéntricos". En Edgardo Lander (ed.). La colonialidad del saber: eurocentrismo y ciencias sociales. Perspectivas latinoamericanas. Buenos Aires: CLACSO, 2000. 
Lander, Edgardo. "Diálogos a través del Atlántico Sur: saberes hegemónicos y saberes alternativos". (c. 2000: 2). Disponible en: <http://bibliotecavirtual. clacso.org.ar/ar/libros/aladaa/lander.rtf>.

Mignolo, Walter. "Herencias coloniales y teorías postcoloniales". En Beatriz González Stephan (ed.). Cultura y Tercer Mundo: 1. Cambios en el Saber Académico. Caracas: Nueva Sociedad, 1996.

Mignolo, Walter. "Posoccidentalismo: las epistemologías fronterizas y el dilema de los estudios (latinoamericanos) de área”, Revista Iberoamericana LXII/176177, julio-diciembre, 1996.

Negre Rigol, Pedro. Sociología del Tercer Mundo. Una introducción a sus problemas sociológicos. Buenos Aires: Paidós, 1975.

Pletsch, Carl. "The Three Worlds, or the Division of Social Scientific Labor, circa 1950-1975”, Comparative Study of Society and History 23/4, octubre, 1981.

Santos, Boaventura De Sousa. Una epistemología del Sur: la reinvención del conocimiento y la emancipación social. México: Siglo XXI-CLACSO, 2009. 